\title{
Effect of the radial electric field on the fluctuation-produced transport in the $\mathrm{H}-1$ heliac
}

\author{
M G Shats \\ Plasma Research Laboratory, Research School of Physical Sciences and Engineering, Australian \\ National University, Canberra, ACT 0200, Australia \\ E-mail: michael.shats@anu.edu.au
}

Received 12 May 1999, in final form 26 July 1999

\begin{abstract}
Highly coherent low-frequency fluctuations are studied experimentally in the low electron temperature plasma in the H-1 toroidal heliac. The fluctuations, presumably pressuregradient-driven resistive MHD modes, produce considerable radial particle transport. A build-up of strong sheared radial electric field leads to dramatic modifications in the fluctuation-driven transport. These modifications correlate with sudden changes in the plasma confinement, resembling low-tohigh transitions in other machines. Strong negative shear in the radial electric field eventually leads to the suppression of the fluctuations, while the onset of the strong positive shear correlates with the reversal of the fluctuation-driven particle flux, leading to the inward-directed pinch. It is concluded that the radial electric field modifies radial profiles of the fluctuation propagation velocity due to the Doppler shift, and it is this velocity shear which is important for the transport modifications.
\end{abstract}

\section{Introduction}

Understanding modifications in the fluctuation-induced transport during transitions to improved confinement modes in magnetized plasmas is one of the key steps towards using high confinement in a controllable way. It is believed that the modifications of turbulence, and the anomalous transport associated with it, is responsible for the confinement improvement [1]. The most viable model of such a modification is that the $E \times B$ sheared plasma flow suppresses turbulence and fluctuation-driven transport [2,3]. This idea has been developed from both theoretical work (see, for example, [1] and references therein, and [4-6]) and experimental observations of the fluctuation level reduction at both the edge and internal transport barriers in tokamaks [7-12].

Though the fluctuation suppression is often observed during the transitions to improved confinement modes, there is growing evidence that the reduction in the fluctuation level is not the only possible mechanism of the anomalous transport modification. The reduction of the turbulence-driven particle flux due to the change in the relative phase of the pressure and potential fluctuations has been shown in numerical simulations [13] and analytically [14]. In the edge plasma region of the DIII-D tokamak, it has been found that in some cases, fluctuation levels are increased at the edge transport barrier but the transport is reduced due to the change in the cross phase between density and potential fluctuations [15]. It has also been shown that the turbulent fluxes have an intermittent character in the low-confinement mode in tokamaks and stellarators $[16,17]$, and that the time-averaged flux can even radially reverse at the plasma edge to become inward-directed, as observed in the TEXTOR-94 tokamak [18]. 
In the H-1 heliac we observe two improved confinement modes which differ by the fluctuation behaviour across the transition to the higher confinement. In the so-called 'quiescent' high mode $\left(\mathrm{H}_{\mathrm{q}}\right.$ mode $)$ the fluctuation level drops by more than an order of magnitude compared to the low mode [19]. Fluctuations persist after transitions to the 'fluctuating' high mode $\left(\mathrm{H}_{\mathrm{fl}}\right.$ mode), but modifications in the fluctuation cross phase lead to the radial reversal of $\Gamma_{\mathrm{fl}}=\left\langle\tilde{n} \tilde{E}_{\mathrm{pol}}\right\rangle / B$ from outward- to inward-directed, an inward convective pinch [20]. Such flux reversal has been shown to be symmetric on the flux surfaces, leading to the improvement in the particle confinement. Since the plasma parameters at which these two high-confinement modes are observed are similar, it would be very useful to analyse the details of the conditions at which one or another mode is reached as this may shed some light on the physics of the fluctuation modifications.

In this paper we analyse experimental results on the plasma fluctuations in the $\mathrm{H}-1$ heliac in order to study effects of the radial electric field on the fluctuations, and analyse the conditions at which fluctuation-driven transport is modified in either $\mathrm{H}_{\mathrm{q}}$ or $\mathrm{H}_{\mathrm{fl}}$ modes.

\section{Confinement transitions in the $\mathrm{H}-1$ and experimental set-up}

The $\mathrm{H}-1$ heliac is a 3-field period helical axis stellarator [21] with a major radius of $R=1.0 \mathrm{~m}$ and an average minor radius of $\langle a\rangle=0.2 \mathrm{~m}$. The magnetic field structure of H-1 is characterized by a relatively high rotational transform $(t=1.1-1.5$ in the described experiments) and a very low global magnetic shear $(\hat{s}=(\rho / t)(\mathrm{d} t / \mathrm{d} \rho) \approx 0.005-0.01)$.

Confinement transitions are observed in the H-1 heliac at low magnetic fields $(<0.15 \mathrm{~T})$, moderate heating power $(<100 \mathrm{~kW}$ of $\mathrm{RF}$ at $7 \mathrm{MHz}$ ), and relatively high ion temperatures (up to $150 \mathrm{eV}$ ) [19,22]. In the discharges in heavy gases (argon, neon, helium), line radiation dominates the energy confinement and limits the electron temperature to less than $20-30 \mathrm{eV}$ in argon and less than $40-60 \mathrm{eV}$ in helium plasma. In these conditions probes can be used in the inner plasma. Previous studies in the H-1 [19,22] have shown that:

(i) close to the critical plasma conditions the density and ion temperature spontaneously increase across the transition by $50-100 \%$ in about $1 \mathrm{~ms}$;

(ii) the radial electric field doubles in the outer plasma region $(r / a=0.7-1.0)$;

(iii) transitions are correlated with the reduction in the fluctuation level and the turbulent transport;

(iv) transitions to improved confinement are not correlated with any significant changes in the ion poloidal or toroidal rotation velocities, which were found to be about ten times lower than the corresponding $E \times B$ drift velocities.

It has also been found [23], that driving plasma up to a bifurcation point by changing any of the macroscopic parameters (RF power, magnetic field or the neutral particle density) always leads to a systematic increase in the negative radial electric field before the transition, up to some critical value $E_{\mathrm{r}}=E_{\mathrm{cr}}$. After the transition, $E_{\mathrm{r}}$ increases further in the H-mode due to steeper ion pressure profiles. A qualitative picture of the $E_{\mathrm{r}}$ formation has been presented in [23]. It has been shown that the relative ion-electron loss in the $\mathrm{H}-1$ can explain various scenarios of the confinement bifurcations, including low-to-high $(\mathrm{L}-\mathrm{H})$ and high-to-low $(\mathrm{H}-\mathrm{L})$ transitions.

Since transitions are observed at low $T_{\mathrm{e}}$, a number of probe diagnostics have been used in the inner regions of plasma [24]. Visible spectroscopy has also been used in these experiments [22] for the neutral particle characterization. In this paper we describe experimental results on the characterization of the fluctuations in the low-confinement mode (L-mode), and study the correlation of the turbulence modifications with the evolution in the radial electric field. 
In these experiments we use several combinations of the triple probes, described in references $[20,23,24]$.

\section{Fluctuations in low confinement mode}

Fluctuations in the L-mode exhibit many features which are independent of the magnetic configuration, the RF power, or the ion mass of the working gas. In this section we analyse results obtained in argon discharges in two of the most well-explored magnetic configurations: one having the largest plasma volume and a rotational transform of $t(\rho)=1 / q=1.1-1.2$ (standard configuration), and the other, which is characterized by the lowest magnetic shear and a rotational transform of $t=1 / q \approx 1.41$ (where $q$ is a safety factor).

Figures $1(a)$ and $1(b)$ are the frequency spectra of the ion saturation current and the floating potential correspondingly measured using a triple probe at $r / a=0.8$. Figure $1(c)$ shows the spectrum of the radial component of the magnetic fluctuations measured using a pick-up coil located about $3 \mathrm{~cm}$ outside the last flux surface. All three spectra show that fluctuations are dominated by a strong harmonic at the frequency of about $5 \mathrm{kHz}$. The frequency of this harmonic changes with the plasma parameters, as discussed later in this section. The crosscorrelation between $\tilde{I}_{\mathrm{s}}$ and $\tilde{B}_{\mathrm{r}}$ is high $(>0.7)$ and it decreases as the electrostatic probe is moved further inside the plasma. The multiple frequency harmonics seen by the electrostatic probe are also observed by the magnetic probe.

A poloidal wavenumber of the fluctuations is measured using a fork probe: two triple probes separated poloidally by $\Delta y=15 \mathrm{~mm}$. The distance between the probes is chosen to be large enough for unambiguous determination of the phase shift $\Delta \varphi$, from which the wavenumber is determined as $k_{\mathrm{pol}}=\Delta \varphi / \Delta y$. Shown in figure 2 are the radial profiles of the measured poloidal wavenumber of the fluctuations of the ion saturation current (full diamonds) and of the estimated poloidal wavenumber $k=m / r$, assuming the poloidal mode number is equal to $m=1$ (open squares). The observation of the $m=1$ mode in the standard magnetic configuration has also been confirmed by spectroscopic measurements [25]. In the magnetic configuration with higher rotational transform $(1 / q>1.4)$ the poloidal mode number is typically higher $(m=2)$. The modes propagate poloidally in the direction of the $E \times B$ drift, which coincides with the direction of the electron diamagnetic drift velocity in the $\mathrm{H}-1$. The poloidal phase velocity of the fluctuations is very close to the $V_{E \times B}$ drift velocity as shown in figure 3. This $E \times B$ drift velocity is computed using the measured plasma potential profile.

A toroidal mode number, determined using two triple probes separated toroidally by $\Delta z \approx 0.18 \mathrm{~m}$, is equal to $n=3$ or 6 in the L-mode. This $n$ is a multiple of the number of magnetic field periods in the $\mathrm{H}-1$ (three): one or two fluctuation wavelengths per period.

The radial structure of the mode has been studied by measuring the phase shift between two radially separated probes: one fixed at the edge and another scanned through the plasma radius. The results of these measurements are shown in figure 4. The radial structure of the fluctuations exhibits a mixture of $l=1$ radial mode number, which is dominant in the fluctuations of the ion saturation current (phase shift of about $\pi$ along the plasma radius), as shown in figure $4(a)$, and $l=0$ (phase shift of about $\pi / 2$ along the plasma radius), which dominates the plasma potential fluctuations as shown in figure $4(b)$.

The maximum level of the density fluctuations $\left\langle\tilde{I}_{\mathrm{s}}\right\rangle$ coincides with the radial region of the highest pressure gradient, as shown in figure 5 , while the potential fluctuations $\tilde{V}_{\mathrm{p}}$ have an extra maximum at about the magnetic axis. This is consistent with the measurements of the radial mode number $(l)$ mentioned above.

The frequency of the strongest frequency harmonic increases with both the RF power and the magnetic field. The frequency dependence on $P_{\mathrm{RF}}$ for a fixed magnetic field is shown in 


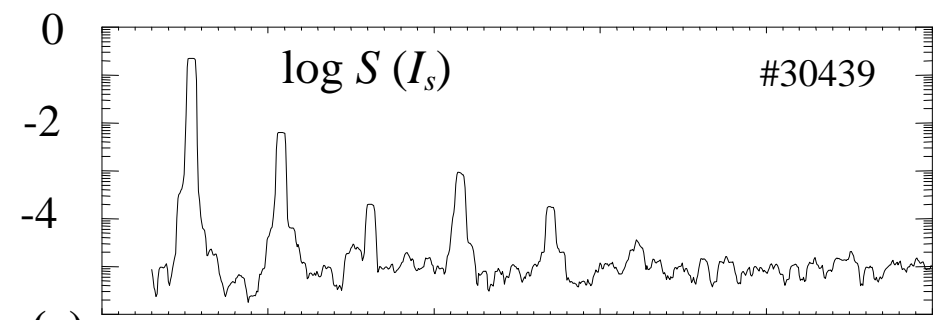

(a)
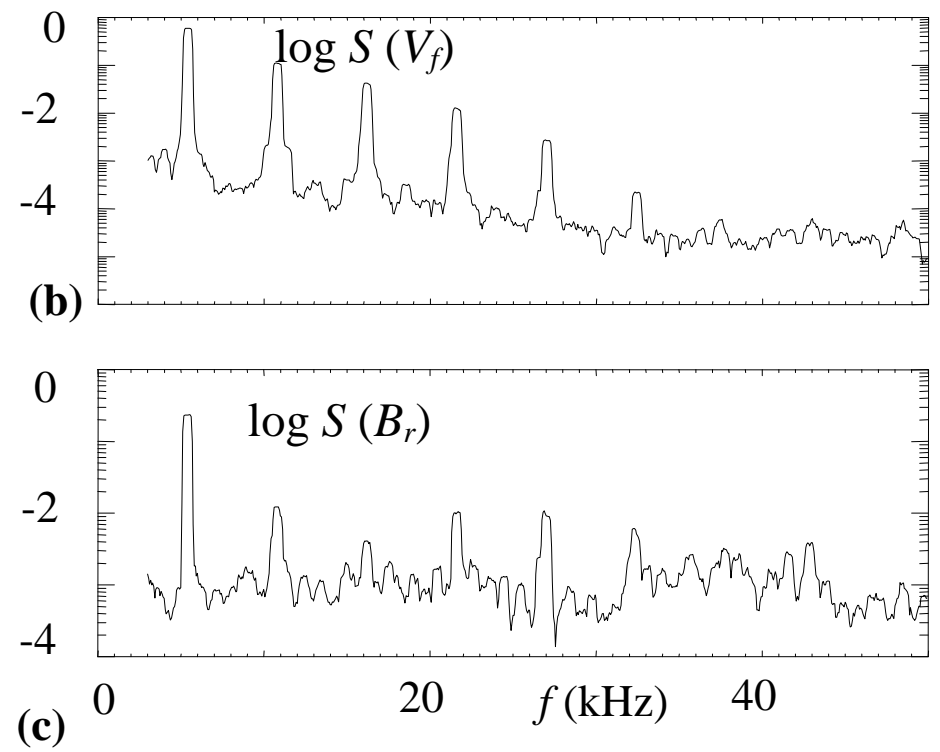

Figure 1. Frequency spectra of the fluctuations in the ion saturation current $(a)$, floating potential $(b)$ and magnetic field $(c)$ in low-confinement mode. The triple probe is located at $r / a \approx 0.8$ and magnetic probe is at $r / a \approx 1.3$.

figure 6, together with the corresponding change in the average radial electric field. It has been shown in [23] that in the described magnetic configuration the increase in $P_{\mathrm{RF}}$ leads to a higher ion temperature $T_{\mathrm{i}}$, higher ion loss from the inner plasma regions and, as a result, a higher $E_{\mathrm{r}}$. Similarly, the increase in magnetic field leads to a higher $E_{\mathrm{r}}$ and eventually, when $E_{\mathrm{r}}$ reaches some critical value $E_{\mathrm{r}} \approx E_{\mathrm{cr}}$, transitions to improved confinement are observed. Figure 7 shows the parallel phase velocity of the fluctuations $V_{\| \mathrm{ph}}=\omega / k_{\|}$, the ion thermal velocity $V_{\mathrm{ti}}$, and the $E \times B$ drift velocity $V_{E \times B}=E_{\mathrm{r}} / B$, versus the magnetic field at constant $\mathrm{RF}$ power. It is seen that the fluctuation parallel phase velocity is well correlated with the $E \times B$ drift velocity. Also, the fluctuation parallel phase velocity is lower than the ion thermal velocity. The latter observation is important for the identification of the underlying instability and will be discussed in section 5 .

The parallel phase velocity of the fluctuations is estimated as

$$
V_{\| \mathrm{ph}}=\omega / k_{\|}=(2 \pi f \Delta z) / \Delta \varphi,
$$

where $f$ is the frequency of the first harmonic of the density fluctuations, $\Delta z$ is the distance between the two toroidally separated probes described above $(\Delta z \approx 0.18 \mathrm{~m})$, and $\Delta \varphi$ is the measured phase shift between the two probe signals at frequency $f$. The probes are aligned to the magnetic field line as follows. One of the probes is fixed at $r / a \sim 0.5$ (where the 


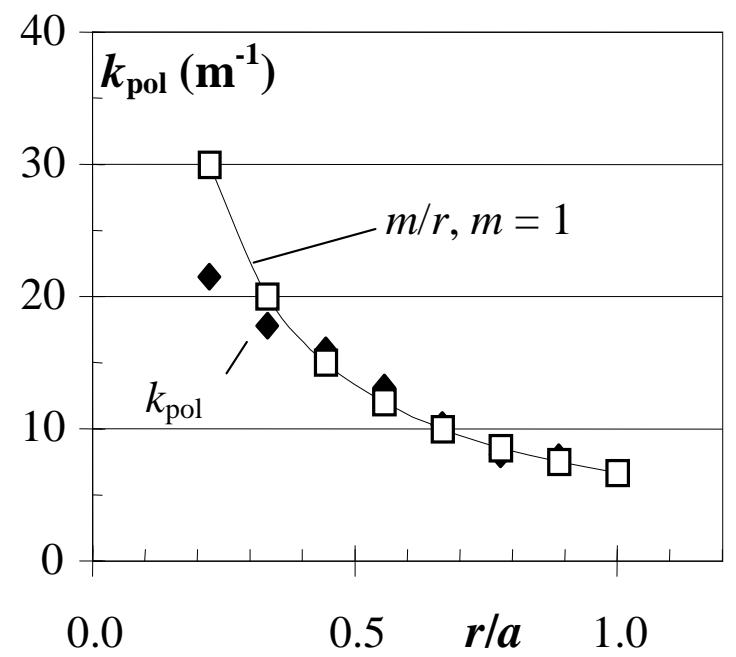

Figure 2. Radial profile of the experimentally measured poloidal Wavenumber of the density fluctuation (full diamonds) and computed poloidal mode number assuming $m=1$ mode (open squares).

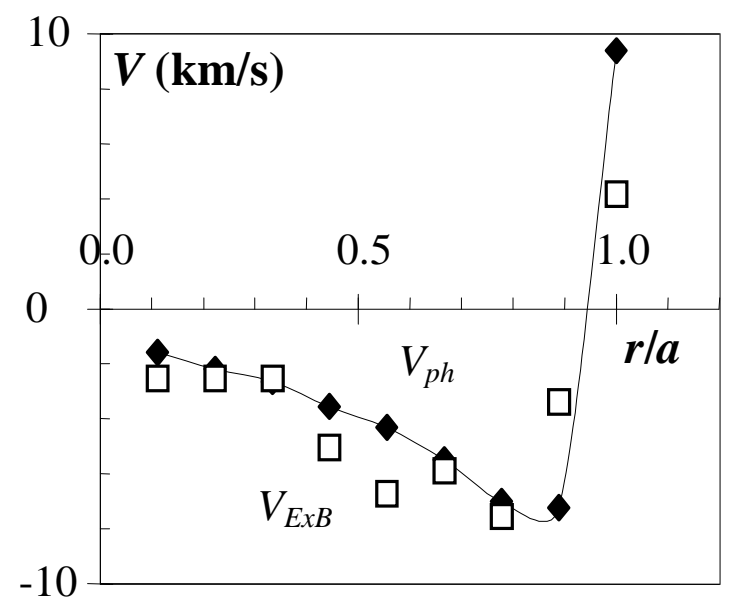

Figure 3. Radial profile of the experimentally measured poloidal phase velocity of the density fluctuation (full diamonds) and poloidal component of the $E \times B$ drift velocity estimated from the measured radial electric field profile (open squares).

fluctuation amplitude is highest), while the other one is moved in the poloidal plane until the cross-correlation between the two signals reaches its maximum $(\sim 0.98)$.

\section{Fluctuation modifications during transitions to high-confinement modes}

The dynamics of the transitions from the L- to H-mode are studied during the power step discharges described in [23]. The RF power is increased in four steps from 0 to $\sim 90 \mathrm{~kW}$ during the plasma discharge shown in figure 8. During the first three steps the plasma is in the L-mode and it is dominated by the low mode number fluctuations described in section 3 . At about $60 \mathrm{~ms}$, a small increase in the RF power triggers the transition to the 'quiescent' $\mathrm{H}$-mode. 


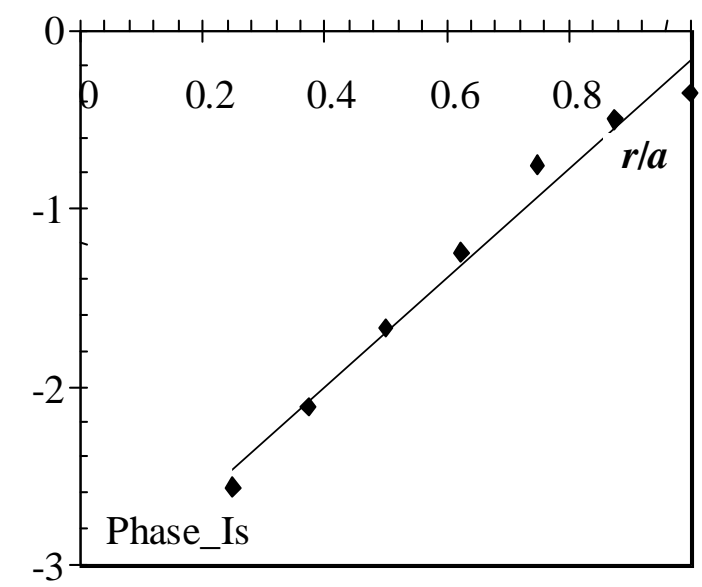

(a)

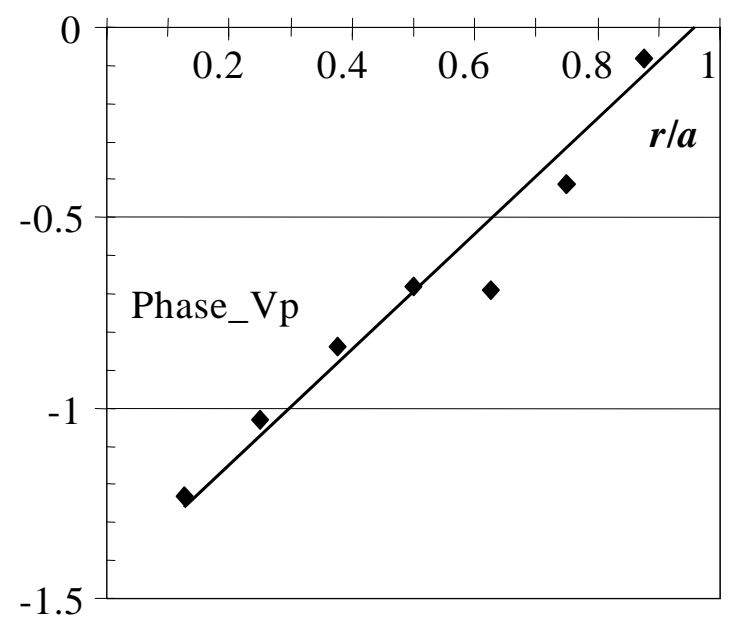

(b)

Figure 4. Radial mode structure of the fluctuations in the ion saturation current $(a)$ and in the plasma potential $(b)$.

The electron density (actually, the ion saturation current at $r / a=0.5$ ) nearly doubles across the transition, while the density fluctuation level drops by about one order of magnitude as shown in figures $8(b)$ and $(c)$. Fluctuations of the plasma potential are suppressed as well. This leads to about a 100 times reduction in the outward-directed fluctuation-driven radial particle flux and confinement improves [19].

An example of a transition to the fluctuating high mode is illustrated in figure 9 for a power step discharge very similar to that just described. The conditions are nearly the same but the neutral gas filling pressure and the magnetic field are slightly lower compared to the conditions of figure 8 . The transition to the $\mathrm{H}_{\mathrm{f}}$-mode occurs in the middle of the third power step at $t \sim 53 \mathrm{~ms}$ and it is followed by a two-fold increase in density. The main difference with figure 8 is that the density (and the plasma potential) fluctuations are not suppressed after the transition. The fluctuation level remains high $\left.\left(\left\langle\tilde{I}_{\mathrm{s}}\right\rangle / I_{\mathrm{s}}\right\rangle>6 \%\right)$ in the $\mathrm{H}_{\mathrm{ff}}-$ mode. It has been shown in [20] that the fluctuation-driven radial particle flux reverses across the transition to 


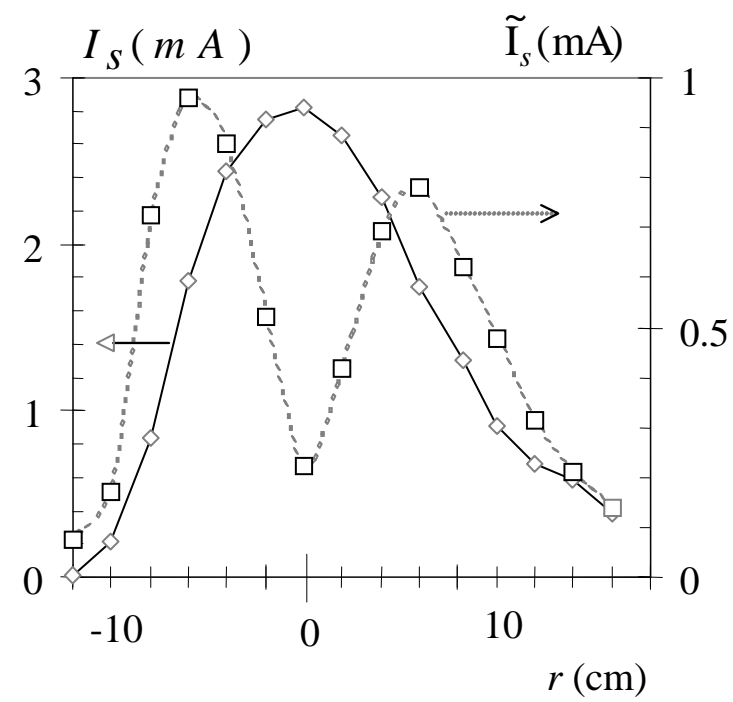

Figure 5. Radial profile of the ion saturation current (full diamonds) and its fluctuations (open squares) in the low-confinement mode.

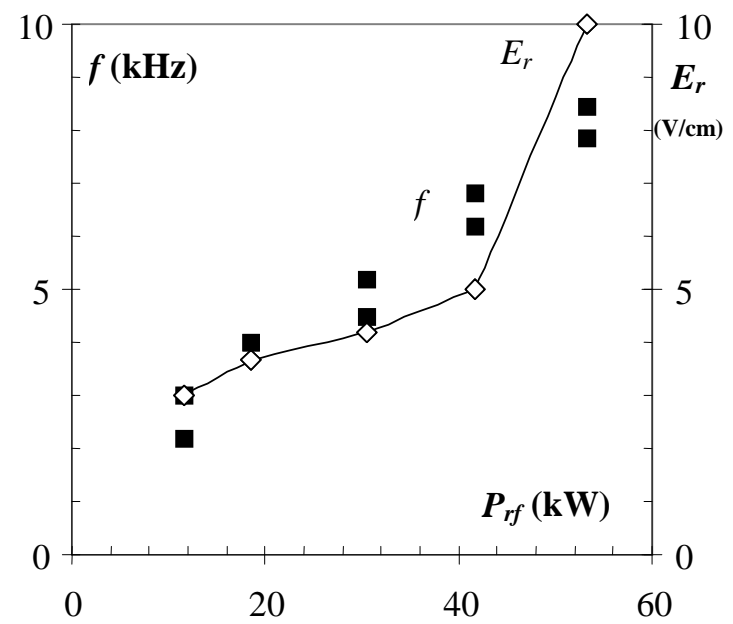

Figure 6. Frequency of the strongest fluctuation harmonic (full squares) and the radial electric field (open diamonds) versus RF power at fixed magnetic field.

the fluctuating H-mode. This reversal of the flux, defined as $\Gamma_{\mathrm{fl}}=\left\langle\tilde{n} \tilde{E}_{\mathrm{p}}\right\rangle / B$, is principally due to a change of sign of the phase shift between the density and potential fluctuations.

We compare radial profiles of the plasma parameters in these three confinement modes shown in figures 8 and 9, namely, the low mode $(t=(35-40) \mathrm{ms}$ in figures 8 and 9), quiescent high mode $(t=(65-70) \mathrm{ms}$ in figure 8$)$, and fluctuating high mode $(t=(65-70) \mathrm{ms}$ in figure 9$)$. The radial profiles of the plasma parameters in these three modes are shown in figure 10. These profiles were obtained on a shot-to-shot basis by radially moving a tripleprobe assembly. The assembly consists of two triple-probes radially separated by $15 \mathrm{~mm} \mathrm{[23].}$ The radial electric field is deduced from a two-point measurement of the plasma potential, $E_{\mathrm{r}}=-\left(V_{\mathrm{r}_{2}}-V_{\mathrm{r}_{1}}\right) /\left(r_{2}-r_{1}\right)$, where $\left(r_{2}-r_{1}\right)=15 \mathrm{~mm}$. Thus, the measured $E_{\mathrm{r}}$ profile has 


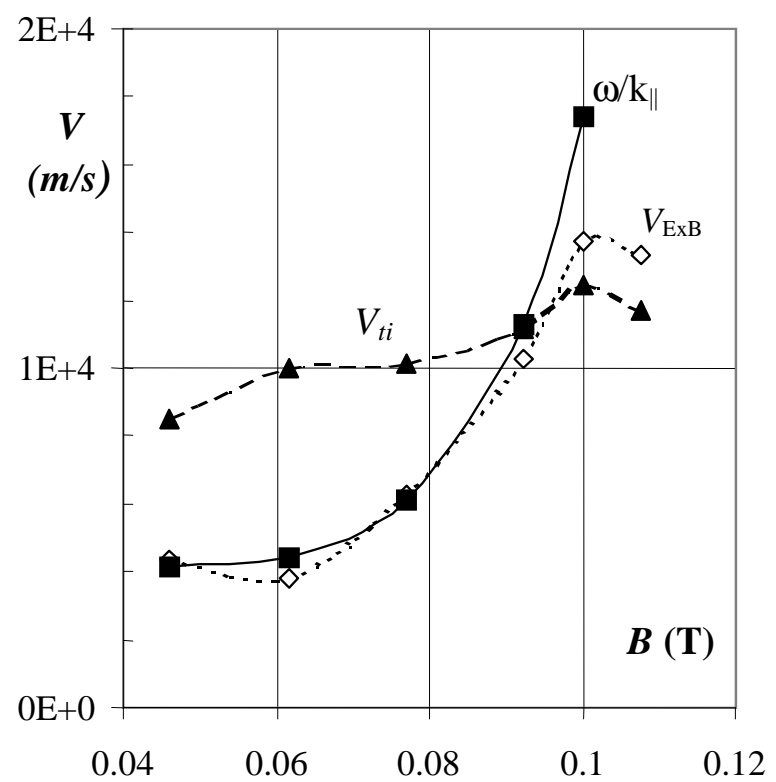

Figure 7. $E \times B$ drift velocity (open diamonds), parallel phase velocity of the fluctuations $V_{\mathrm{ph}}=\omega / k_{\|}$(full squares) and ion thermal velocity $V_{\mathrm{ti}}$ (full triangles) versus the magnetic field.

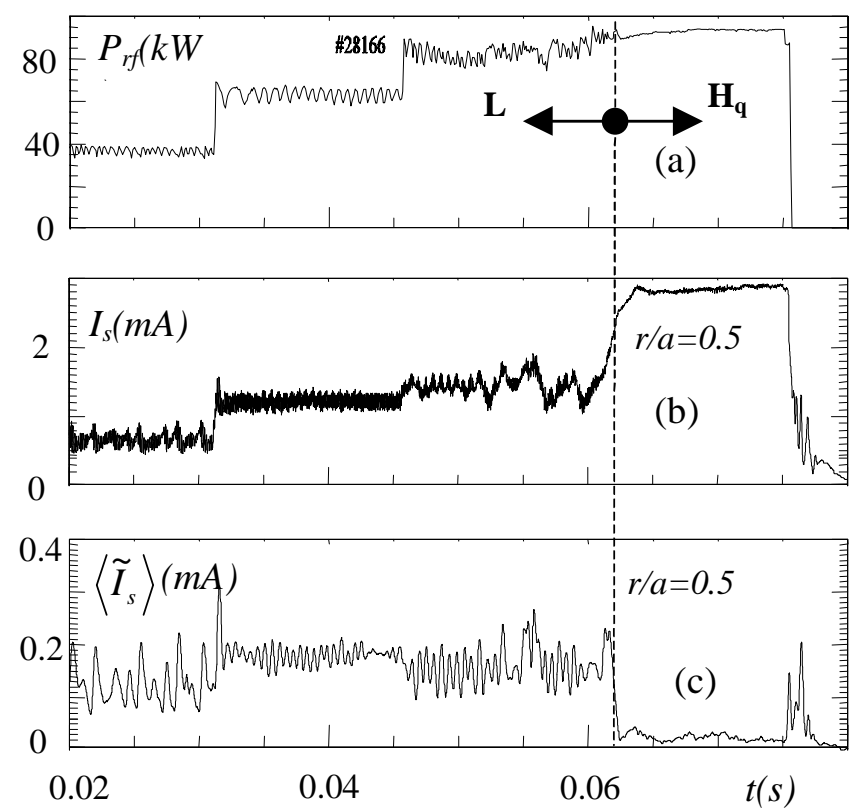

Figure 8. Time evolution of the RF power $(a)$, ion saturation current at $r / a=0.5(b)$ and fluctuations in the ion saturation current at $r / a=0.5(c)$ during discharge with the transition to the 'quiescent' H-mode.

been cross-checked by comparison with the differentiated plasma potential profile obtained using a single triple-probe. Radial profiles of the plasma parameters have also been measured several times to check for reproducibility. A relative error for the profiles of figure 10 has been 


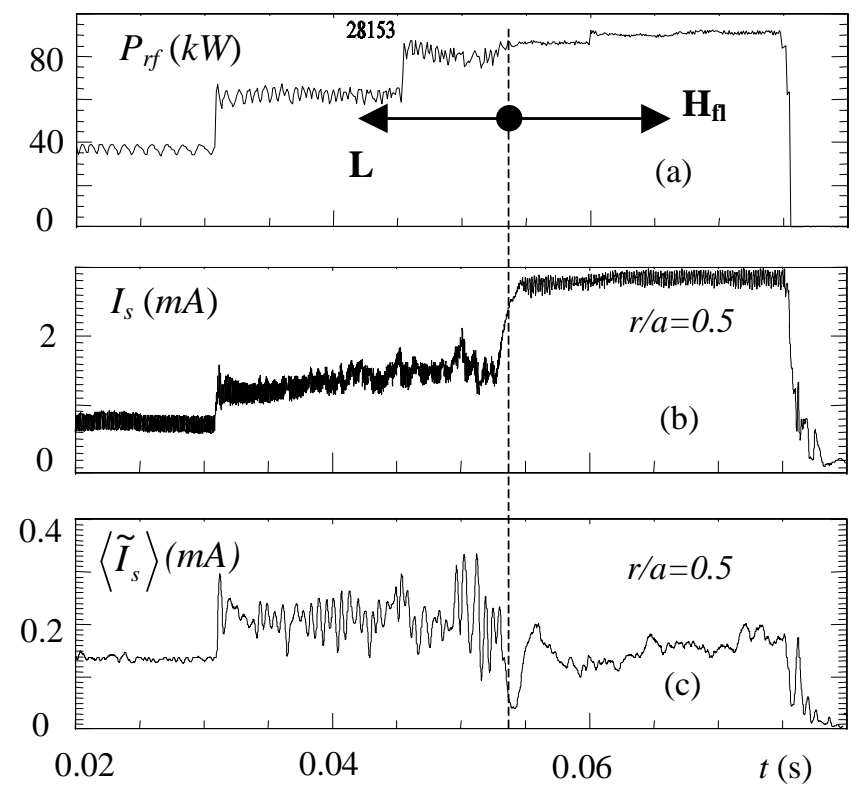

Figure 9. Time evolution of the RF power $(a)$, ion saturation current at $r / a=0.5(b)$ and fluctuations in the ion saturation current at $r / a=0.5(c)$ during discharge with the transition to 'quiescent' H-mode.

found to be less than $\pm 10 \%$ in the radial region of $r / a \geqslant 0.5$. The insertion of two triple-probes in the inner plasma region $(r / a<0.5)$ leads to the plasma perturbation and larger errors.

The plasma density, proportional to the ion saturation current (figure 10(a)), almost doubles across the transition from the $\mathrm{L}$ - to either the $\mathrm{H}_{\mathrm{q}}$ - or $\mathrm{H}_{\mathrm{fl}}$-mode at $r / a \approx 0.5$, while at the outer quarter of the plasma radius $(r / a \geqslant 0.75)$ it is practically unchanged. The density profiles in the two high-confinement modes are similar, but the maximum density in the $\mathrm{H}_{\mathrm{fl}}$ mode is somewhat higher.

The density fluctuation level is modified differently across transitions from the $\mathrm{L}-$ to $\mathrm{H}_{\mathrm{q}}$ or $\mathrm{H}_{\mathrm{fl}}$-modes as shown in figure $10(b)$. Fluctuations in the $\mathrm{H}_{\mathrm{q}}$-mode are reduced everywhere inside the last closed flux surface by nearly an order of magnitude. In the $\mathrm{H}_{\mathrm{fl}}$-mode such a dramatic reduction in the fluctuation level is observed only at $r / a>0.8$, while at $r / a \leqslant 0.75$ the fluctuation reduction is relatively modest $\left(\left\langle\tilde{I}_{\mathrm{s}}\right\rangle /\left\langle I_{\mathrm{s}}\right\rangle \sim 7 \%\right.$ at $\left.r / a=0.5\right)$.

The radial electric field profile in the L-mode (figure $10(c)$ ) is rather featureless with $E_{\mathrm{r}}$ being in the range between 0 and $-7 \mathrm{~V} \mathrm{~cm}^{-1}$. In the quiescent $\mathrm{H}_{\mathrm{q}}$-mode $E_{\mathrm{r}}$ becomes more negative, reaching $-32 \mathrm{~V} \mathrm{~cm}^{-1}$ near the plasma edge. The radial electric field profile in this regime is characterized by a negative $E_{\mathrm{r}}$ shear of about $\mathrm{d} E_{\mathrm{r}} / \mathrm{d} r \approx-12 \mathrm{~V} \mathrm{~cm}^{-2}$. In contrast, the $E_{\mathrm{r}}$ profile in the $\mathrm{H}_{\mathrm{fl}}$-mode exhibits a clear reversal in the radial electric field shear of $\mathrm{d} E_{\mathrm{r}} / \mathrm{d} r$ at $r / a \approx 0.8$. It should be noted that in the $\mathrm{H}_{\mathrm{fl}}$-mode, fluctuations are suppressed in the region of negative $E_{\mathrm{r}}$ shear at $r / a>0.8$, where $\mathrm{d} E_{\mathrm{r}} / \mathrm{d} r \approx-14 \mathrm{~V} \mathrm{~cm}^{-2}$, while in the region where $\mathrm{d} E_{\mathrm{r}} / \mathrm{d} r>0(0.5 \leqslant r / a \leqslant 0.75)$, the fluctuation intensity is less affected. It is in this region of positive $\mathrm{d} E_{\mathrm{r}} / \mathrm{d} r$ that the phase between the density and potential fluctuations reverses in the $\mathrm{H}_{\mathrm{fl}}$-mode, as shown in figure $10(d)$. The phase reversal is responsible for the radial reversal in the fluctuation-produced particle flux, as reported in [20].

The correlation between the reversal in $\mathrm{d} E_{\mathrm{r}} / \mathrm{d} r$ and the reversal in the phase between $\tilde{E}_{\mathrm{p}}$ and $\tilde{n}$ has been confirmed under several slightly different experimental conditions (varied RF 

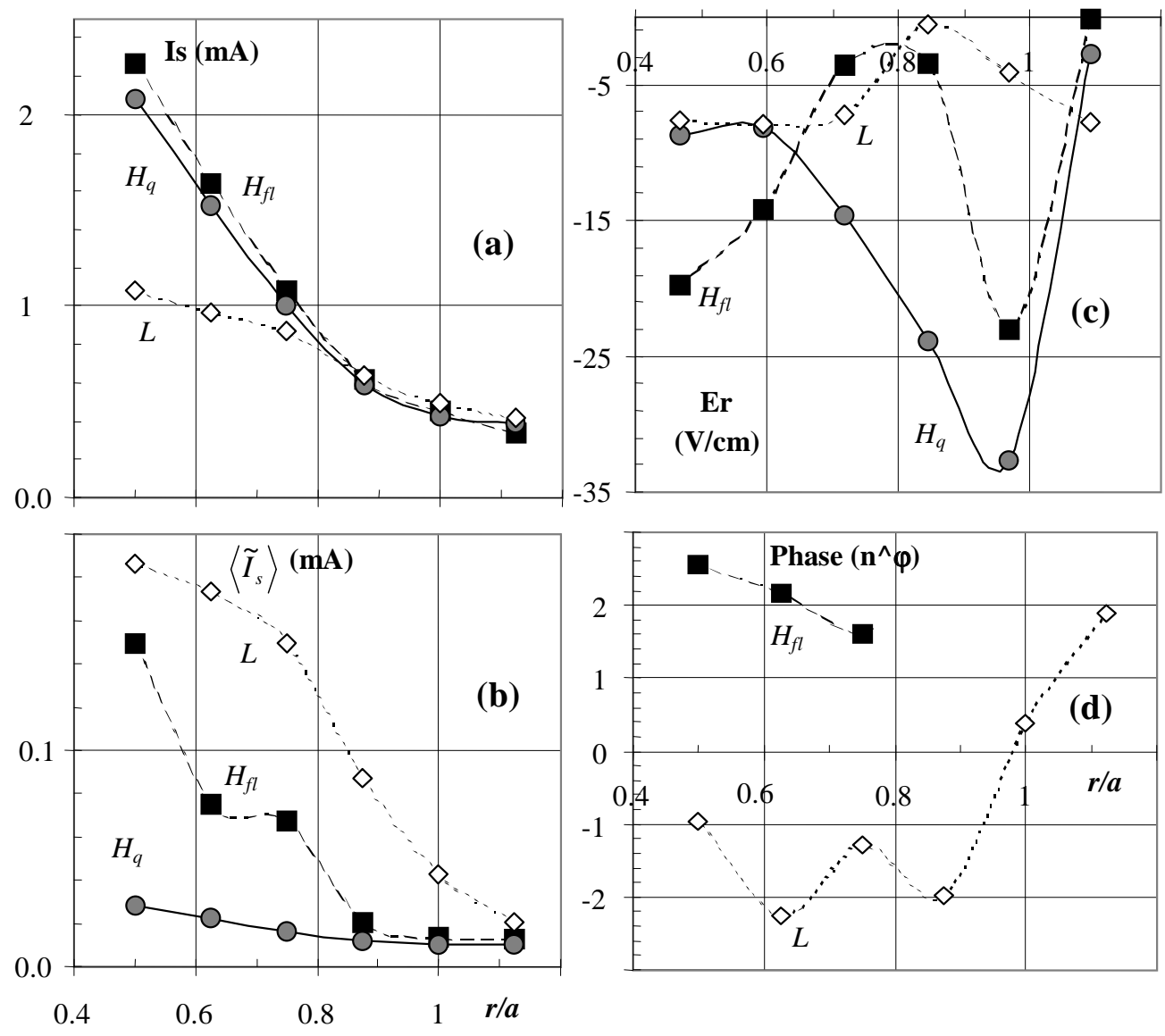

Figure 10. Radial profiles of the plasma parameters in different confinement modes: ion saturation current $(a)$, fluctuations of the ion saturation current $(b)$, radial electric field $(c)$, and phase between density and potential fluctuations $(d)$.

power, magnetic field, filling pressure). The onset of the radial region where $\mathrm{d} E_{\mathrm{r}} / \mathrm{d} r=(+7$ to +12$) \mathrm{V} \mathrm{cm}^{-2}$ is always correlated with $\Gamma_{\mathrm{fl}}$ reversal.

Measurements of the time evolution of the radial electric field during the $\mathrm{L}-\mathrm{H}_{\mathrm{q}}$ and $\mathrm{L}-\mathrm{H}_{\mathrm{fl}}$ transitions have been performed to double check the onset of the positive $E_{\mathrm{r}}$ shear during a single plasma discharge. Three triple-probes were located at $r_{1} / a=0.5, r_{2} / a=0.69$ and $r_{3} / a=0.93$ so that the time evolution of the radial electric field at $r_{12} / a=0.59$ and $r_{23} / a=0.81$ could be measured simultaneously. The results are presented in figures 11 and 12 for the same shots as in figures 8 and 9 . During the $\mathrm{L}-\mathrm{H}_{\mathrm{q}}$ transition (figure 11), the radial electric field becomes more negative at $r / a=0.81$ (figure 11(b)), while at $r / a=0.59$ (figure $11(a)$ ), $E_{\mathrm{r}}$ does not change significantly across the transition in qualitative agreement with the profiles in figure 10. This $E_{\mathrm{r}}$ evolution differs from that during the $\mathrm{L}-\mathrm{H}_{\mathrm{ff}}$ transition (figure 12). The radial electric field at $r / a=0.59$ becomes more negative across the $\mathrm{L}-\mathrm{H}_{\mathrm{fl}}$ transition as shown in figure 12(a), while at $r / a=0.81$ (figure 12(b)) $E_{\mathrm{r}}$ does not change significantly. These measurements also confirm the formation of the positive $\mathrm{d} E_{\mathrm{r}} / \mathrm{d} r$ region in the fluctuating $\mathrm{H}$-mode. 


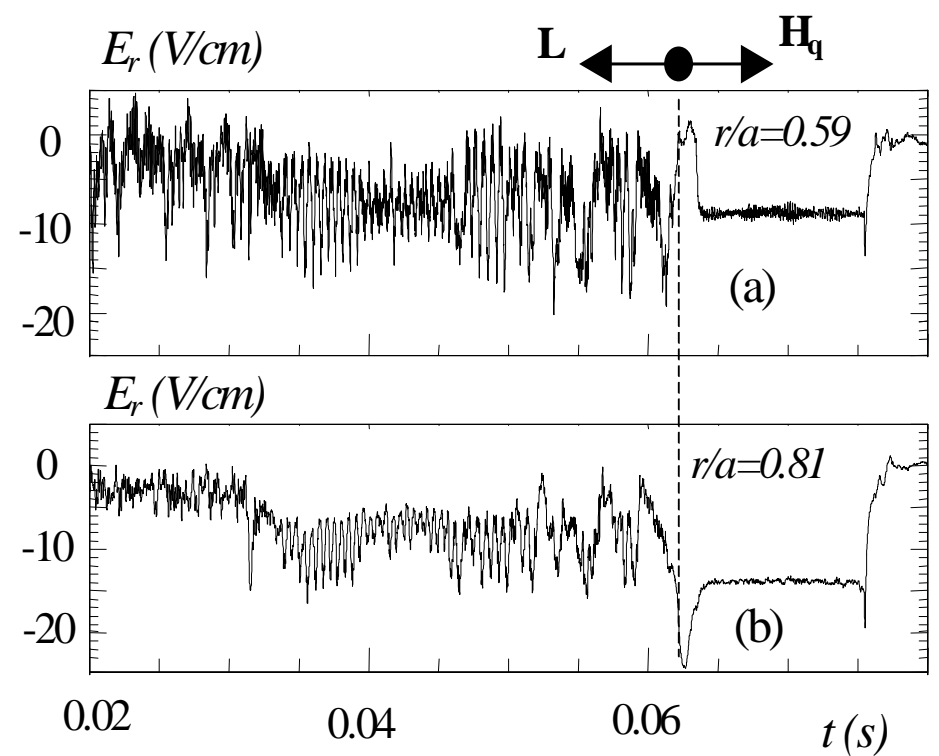

Figure 11. Time evolution of the radial electric field at $r / a=0.59(a)$, and at $r / a=0.81(b)$ in the discharge with the transition to the 'quiescent' H-mode, as in figure 8.

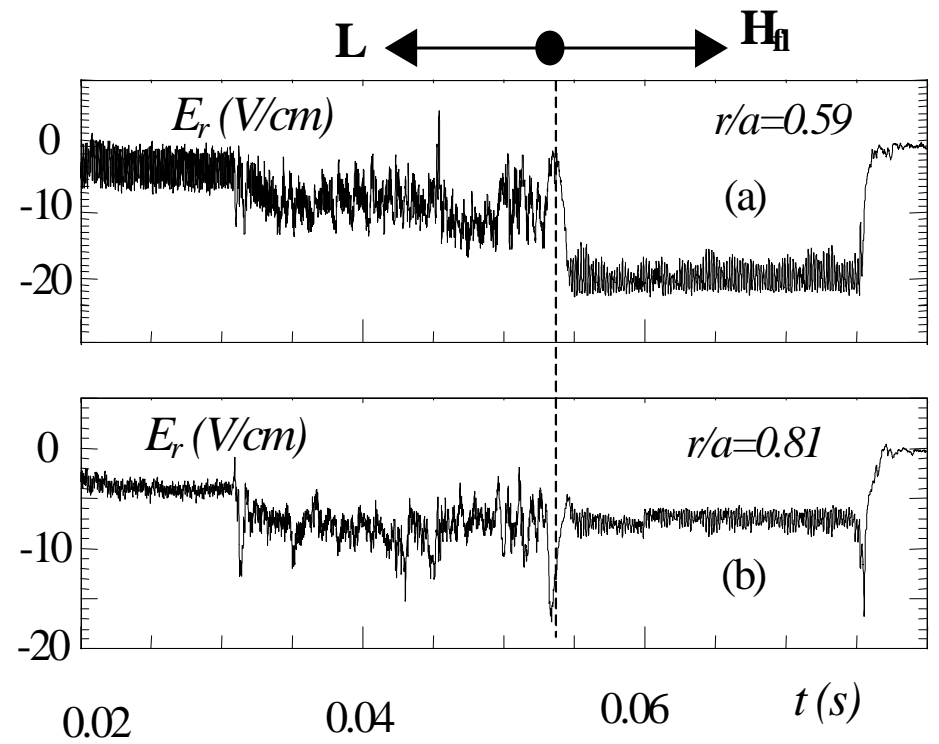

Figure 12. Time evolution of the radial electric field at $r / a=0.59(a)$ and at $r / a=0.81(b)$ in the discharge with the transition to 'fluctuating' H-mode, as in figure 9.

\section{Discussion}

Low-frequency, quasi-coherent fluctuations of the electron density, plasma potential and magnetic field, described in section 3, are responsible for a significant 'anomalous' particle transport in the $\mathrm{H}-1$ heliac in the low-confinement mode. The maximum intensity of the density fluctuations is localized in the region of maximum density (and pressure) gradient, as shown 
in figure 5. The fluctuation level increases with an increase in the pressure gradient in the low mode. This, for example, can be seen in figures 8 and 9, where the increase in RF power at $t<50 \mathrm{~ms}$ increases the density (and its gradient) through higher ionization, leading to an increase in the fluctuation level.

The fluctuation spectra exhibit several frequency harmonics as shown in figure 1. The fluctuations of different plasma parameters (density, temperature, potential, poloidal and radial magnetic field) are highly correlated. The poloidal phase velocity of the $m=1$ mode coincides with the velocity of the poloidal $E \times B$ drift, presumably due to the Doppler shift as shown in figure 3. This presumption agrees also with the result of Figure 6 . The mode frequency is well correlated with the radial electric field during the RF power scan at fixed magnetic field $B$, in agreement with $\omega / k_{\mathrm{pol}} \propto E / B$. The fluctuation propagation velocity is also correlated with $V_{E \times B}$ during the magnetic field scan at constant RF power, as shown in figure 7. A somewhat similar result, i.e. the correlation of the poloidal propagation velocity of the magnetic fluctuations with the $E \times B$ drift velocity, has been observed in the Alcator C-Mod tokamak [26].

A very important observation is that the component of the phase velocity parallel to the magnetic field is smaller than the ion thermal velocity in our experiment, as shown in figure 7. This excludes electrostatic drift wave instabilities from possible candidates for the observed fluctuations, since such waves should be Landau damped on ions (see, for example, [27]). The fluctuation properties described above are reasonably consistent with resistive pressure-gradient-driven modes (e.g. [28-30] and references therein) or non-resonant resistive instabilities [31], which have been shown to be potentially responsible for anomalous transport in stellarators. A detailed comparison with theory is somewhat complicated by the fact that such a theory should include finite Larmor radius effects, absolutely essential in the H-1 (see [23]), as well as radial electric field effects.

Two different improved confinement modes observed in the $\mathrm{H}-1[18,20]$ are characterized by either suppression of the fluctuations ('quiescent' high mode) or reversal of the phase between density and potential fluctuations ('fluctuating' high mode). Two substantially different profiles of the radial electric field correspond to these two regimes. The $\mathrm{H}_{\mathrm{q}}$ mode is characterized by a high shear of the radial electric field (up to $\mathrm{d} E_{\mathrm{r}} / \mathrm{d} r \approx-9 \mathrm{~V} \mathrm{~cm}^{-2}$ ) in the radial region of $r / a=(0.6-0.9)$. This $E_{\mathrm{r}}$ shear produces a shearing decorrelation rate [2] of

$$
\tau_{\mathrm{s}}^{-1}=\left[\frac{k_{\mathrm{pol}} \Delta r_{\mathrm{m}}}{B_{\mathrm{T}}} \frac{\mathrm{d} E}{\mathrm{~d} r}\right] \geqslant 3 \times 10^{5} \mathrm{~s}^{-1}
$$

where $\Delta r_{\mathrm{m}}$ is the radial mode width $\left(0.04-0.05 \mathrm{~m}\right.$ in our case) and $k_{\mathrm{pol}}$ is the poloidal wavenumber $\left(k_{\text {pol }} \sim 10 \mathrm{~m}^{-1}\right.$, as shown in figure 2$) .1 / \tau_{\mathrm{s}}$ is larger than the turbulence decorrelation rate $\tau_{\mathrm{D}}^{-1}=D_{\mathrm{an}} / \Delta r_{\mathrm{m}}^{2} \sim 2 \times 10^{4} \mathrm{~s}^{-1}$, where $D_{\mathrm{an}}=30 \mathrm{~m}^{2} \mathrm{~s}^{-1}$ is the anomalous diffusion coefficient, estimated by comparing outward particle fluxes in low and quiescent high modes. Thus, our results do not contradict the $E_{\mathrm{r}}$ shear suppression models [2-5] in the 'quiescent' high mode. It should be noted that fast oscillations in the radial electric field before the transition to the $\mathrm{H}$-mode (figures 11 and 12) could reduce the effective shearing rate, as discussed by Hahm et al [32]. Using the results of this paper, we estimate that a reduction factor should not exceed 2-3 in our experiment, so that the condition $1 / \tau_{\mathrm{s}} \gg 1 / \tau_{\mathrm{D}}$ is still satisfied just before the transition to the H-mode.

In the 'fluctuating' high mode, the $E_{\mathrm{r}}$ radial profile exhibits a region of positive $E_{\mathrm{r}}$ shear, as shown in figure $10(c)$. This region of $\mathrm{d} E_{\mathrm{r}} / \mathrm{d} r>0$ correlates with the region of the reversal of the phase between density and potential fluctuations, thus affecting the direction of the fluctuation-driven particle flux $\Gamma_{\mathrm{fl}}=\left\langle\tilde{n} \tilde{E}_{\mathrm{pol}}\right\rangle / B$. Radial profiles of $E_{\mathrm{r}}$ in the $\mathrm{H}_{\mathrm{fl}}$-mode always show two peaks as illustrated in figure 10: one peak at $r / a \sim 1.0$, similar to the $\mathrm{H}_{\mathrm{q}}$-mode, and 
another at $r / a \sim 0.5$. Radial profiles of the fluctuation phase velocity in the poloidal direction also show two peaks, corresponding to the two peaks in $E_{\mathrm{r}}$. Thus, the fluctuation propagation in the $\mathrm{H}_{\mathrm{fl}}$-mode is also correlated with $V_{E \times B}$, similar to the L-mode. Note that the plasma fluid velocity in the H-1 is significantly lower than the corresponding $V_{E \times B}$ velocity [22]. We may thus conclude that it is modifications to the fluctuation propagation velocity due to the $E \times B$ electron drift that affect transport properties of the fluctuations, rather than the sheared mass flow. Hutchinson et al [26] have recently made a similar comment analysing transport barrier phenomena in the Alcator C-Mod tokamak.

Different ways of achieving threshold conditions for the fluctuation suppression in $\mathrm{H}-1$ have been analysed in [23]. It has been shown that the variety of transition scenarios in the H-1 can simply be described as different ways of driving $E_{\mathrm{r}}$ and its shear up to some critical value. Now, based on the discussion above, we may speculate that in fact they are just ways of building up a critical shear in the propagation velocity of the fluctuations in the laboratory frame of reference.

Inward-directed, fluctuation-produced particle fluxes have been observed in several tokamak experiments $[18,33]$, both inside and outside the separatrix. Ionization-driven drift wave instabilities have been considered as potential candidates to explain inward-directed particle fluxes [34]. Though ionization in the H-1 occurs in the inner plasma regions [22], introducing a potential drive for the ionization drift waves, there are several counter arguments. Firstly, as discussed above, the observed fluctuations are probably not electrostatic drift waves. Secondly, analysis of the ionization rate based on the multi-chord spectroscopic measurements of the argon neutral line intensities [22] shows that in the radial region of interest $(r / a \sim 0.5)$, the ionization rate does not change or slightly decreases across the transition shown in figure 9 , reducing the drive.

Further experiments, including comparative studies of the fluctuation-driven transport in different machines may shed more light on the physics of inward-directed anomalous transport in toroidal plasmas.

\section{Acknowledgments}

The author would like to thank M Wakatani, K Toi, J H Harris and W Solomon for fruitful discussions of the results, and D L Rudakov and other members of the Plasma Research Laboratory of the Australian National University for their help during this work.

\section{References}

[1] Carreras B A 1997 IEEE Trans. on Plasma Sci. 251281

[2] Biglari H, Diamond P H and Terry P W 1990 Phys. Fluids B 21

[3] Shaing K C, Crume E C Jr and Houlberg W A 1990 Phys. Fluids B 21492

[4] Hahm T S and Burrell K H 1996 Plasma Phys. Control. Fusion 381427

[5] Hahm T S 1997 Phys. Plasmas 44074

[6] Krane B, Christopher I, Shoucri M and Knorr G 1998 Phys. Rev. Lett. 804422

[7] Doyle E J et al 1991 Phys. Fluids B 32300

[8] Rettig C L et al 1993 Nucl. Fusion 33643

[9] Mazzucato E et al 1996 Phys. Rev. Lett. 773145

[10] Rettig C L et al 1997 Phys. Plasmas 44009

[11] Jasmich S, Van Oost G, Weynants R R and Boedo J A 1998 Plasma Phys. Control. Fusion 401105

[12] Burrell K H et al 1998 Plasma Phys. Control. Fusion 401585

[13] Carreras B A et al 1995 Phys. Plasmas 22744

[14] Ware A S et al 1996 Plasma Phys. Control. Fusion 381343

[15] Moyer R A et al 1995 Phys. Plasmas 22397 
[16] Endler M et al 1995 Nucl. Fusion 351307

[17] Carreras B A et al 1996 Phys. Plasmas 32664

[18] Boedo J et al 1998 Czech. J. Phys. 48/S3 99

[19] Shats M G et al 1996 Phys. Rev. Lett. 774190

[20] Shats M G and Rudakov D L 1997 Phys. Rev. Lett. 792690

[21] Hamberger S M et al 1990 Fusion Technol. 17123

[22] Shats M G et al 1997 Phys. Plasmas 43629

[23] Shats M G et al 1998 Phys. Plasmas 52390

[24] Rudakov D L et al 1999 Rev. Sci. Instrum. 70476

[25] Shats M G and Howard J 1997 Fusion Eng. Design 34-35 271

[26] Hutchinson I H 1999 Plasma Phys. Control. Fusion 41 A609

[27] Alexandrov A F, Bogdankevich L S and Rukhadze A A 1984 Principles of Plasma Electrodynamics (Berlin: Springer) $\mathrm{p} 271$

[28] Carreras B A, Garcia L and Diamond P H 1987 Phys. Fluids 301388

[29] Carreras B A, Lynch V E and Garcia L 1991 Phys. Fluids B 31438

[30] Carreras B A, Lynch V E, Garcia L and Diamond P H 1993 Phys. Fluids B 51491

[31] Ichiguchi K, Nakamura Y and Wakatani M 1991 Nucl. Fusion 312073

[32] Hahm T S et al 1999 Phys. Plasmas 6922

[33] Jha R et al 1993 Nucl. Fusion 331201

[34] Leboeuf J-N et al 1991 Phys. Fluids B 32291 